\title{
An adaptive multi-hop routing with IoT abstraction for minimizing delay-node capacity trade-offs in mobile ad-hoc network
}

\author{
Haitham Shiaibth Chasib ${ }^{1}$, Saddam Raheem Salih ${ }^{2}$, Israa Jaber Khalaf Al-Ogaili ${ }^{3}$ \\ ${ }^{1,3}$ Department of Information Technology, General Directorate of Education in Babylon, \\ Ministry of Education in Iraq, Iraq \\ ${ }^{2}$ Department of Information Technologies, Directorate General of Education in Diyala, \\ Ministry of Education in Iraq, Iraq
}

\begin{abstract}
Article Info
\section{Article history:}

Received Jan 2, 2021

Revised May 14, 2021

Accepted May 28, 2021

\section{Keywords:}

IoT

MANET

Neighbor selection

Offloading data

Route discovery

ABSTRACT

Delay and node capacity are incompatible mobile ad hoc constraints because of the network's versatility and self-disciplined design. It is a challenging problem to maximize the trade-off between the above mobility correlation factors. This manuscript proposes an adaptive multi-hop routing (A.M.R.) for mobile ad-hoc network (MANET) to minimize the trade-off by integrating the internet of things (IoT). IoT nodes' smart computing and offloading abilities are extended to ad-hoc nodes to improve routing and transmission. For MANET nodes in route exploration, neighbor selection, and data transmission, the beneficial features of IoT include enhanced decision making. The traditional routing protocols use IoT at the time of the neighbor discovery process in updating the routing table and localization. The harmonizing technologies with their extended support improve the performance of MANETs has been estimated. The proposed method achieves better throughput (14.16 Mbps), delay (0.118), packet drop (126), and overhead (36 packets) when compared to existing methods.
\end{abstract}

This is an open access article under the CC BY-SA license.

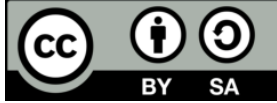

\section{Corresponding Author:}

Haitham Shiaibth Chasib

Department of Information Technology

General Directorate of Education in Babylon

Ministry of Education in Iraq, Iraq

Email: haitham.aljabry2014@gmail.com

\section{INTRODUCTION}

Mobile ad-hoc network (MANET) is an assembly of disparate mobile communicating nodes that interacts in an on-demand fashion. The nodes are autonomous and possess self-configuring, decision making, and routing. MANET exploits different communication and wireless technologies for packet data exchange and information sharing. A MANET node communicates with its neighbor in both single and multi-hop depending on the physical distance of separation. In a multi-hop communication, the sender/the source interacts directly with the neighbor [1], [2] i.e.; the neighbor is present in the line-of-sight of the sender. Instead, in a multi-hop communication, the source/sender banks on a series of neighbors through which the destination node is reached. In an ad-hoc scenario, this reinforces the need for route exploration and neighbourhood extraction [3]. More obviously, in a complex network topology, the nodes require reactive and hybrid routing protocols for route discovery. The routing process is difficult as the protocols must eliminate problems with route discovery, adjust to the behavior of the intrinsic node, and interoperable 
network features. Recent advances in MANET require multi-hop and uninterrupted routing service and transmission functionality to facilitate service efficiency (QoS) [4] for real-time applications.

The design purpose of internet of things (IoT) is to provide stable, reliable, and ubiquitous communication and access to distributed resources. End-users access resources through smart devices such as mobile phones, tablets, digital assistants, etc. through machine interactive applications and services [5], [6]. As IoT incorporates different communication, and storage technologies, it is interoperable with other networks such as ad-hoc, cloud, edge and fog. Resource sharing, decision making, data analytics, access and retrieval, security are some of the features that are inherited from IoT by other interoperable networks. This offers flexible and scalable network configurations of IoT extended to the service of commercial and noncommercial users [7].

Ad-hoc features such as data gathering, information processing, routing decision making, and heterogeneous network communications are inherited by the IoT systems. IoT systems rely on ad-hoc features for route discovery and data gathering [8]. The dual characteristic of the MANET node, i.e., the node can act as a host, and a router is utilized by the IoT for service provisioning and user access. MANET-IoT systems provide composite routing solutions for service discovery and providing multi-level application support. MANET nodes are equipped with smart computational abilities that help to form the core components of IoT [9], [10]. The interoperable nature of IoT aids MANET integration at ease intending to support large-scale smart applications. MANET's general issues and challenges are resolved by the intelligent computing and communication characteristics of IoT systems [10]-[12]. The main contribution of the proposed work is described as follows:

a) Designing MANET's adaptive multi-hop routing (A.M.R.) to reduce the trade-off by incorporating the internet of things (IoT) benefits.

b) To enhance routing and transmission, the smart computing and offloading capacities of IoT nodes are enhanced to ad-hoc nodes

c) The throughput, delay, packet drop, and overhead have been analyzed in the performance evaluation section.

\section{RELATED WORK}

This routing achieves less packet drop and throughput and better energy efficiency by retaining high node energy. A delay concentric multicast routing protocol for MANETs is designed by Chen et al. [13] for improving the node capacity for improving packet admission rate under controlled delay and overhead. The multicast routing tree is constructed by augmenting delay computed one-hop nodes as a measure of controlling delay. By computing the radio channel utilization rate, the transmission rate of the nodes is dynamically varied to sustain varying traffic. Li et al. [14] designed a cluster-induced routing algorithm for improving the performance of IoT harmonized mesh networks. This clustering algorithm accounts interference and network load for evading the routing and transmission constraints. Delay and transmission quality model of the clustering algorithm balances effectively between the mesh node lifetime and IoT traffic sources. This algorithm exploits the buffer and channel interference information of the mesh networks to improve network throughput and reduce packet loss and overhead. O.R.G.M.A. is an opportunistic gradient based forwarding protocol designed by Kang et al. [15], Talib et al. [16] for improving MANET performance. In this routing, the receiver performs routing decisions after receiving the broadcast control message from the sender. Cost management and signal-to-noise ratio (SNR) are exploited in the forwarding method for improving packet delivery ratio and network throughput. Zhang et al. [17] presented a bioinspired hybrid trusted routing protocol (B-iHTRP) for improving the autonomous optimization of MANET. The hybrid protocol integrates ant colony optimization (A.C.O.) and physarum autonomic optimization (P.A.O.) methods. A.C.O. is responsible for segregating the network into zones and identifying the possible routes to the destination. P.A.O. is responsible for deciding routes to satisfy multi-regional communication. This hybrid protocol achieves better delivery ratio and delay for varying node speed and density. Synchronized fuzzy ant system (SynFAnt) is developed by Kacem et al. [18] for route discovery and decision making in ad-hoc networks. This system inherits the benefits of A.C.O., fuzzy transition and Petri nets for improving the routing decisions of MANETs. A.C.O. process addresses the uncertainty in routing decisions. SynFAnt leverages network outcome estimated in terms of packet delivery ratio, delay, and packet acceptance rate and network throughput. Bai et al. [19] introduced constructive relay-based cooperative routing (C.R.C.P.R.) for levitating profitable outcome of MANETs. This relay based routing is designed to mitigate the drawbacks caused due to varying node mobility. It resolves the routing issues by exploiting the neighbor information from the cooperative and relay table of the nodes [20].

Similarly, the routes are adapted based on energy utilization, conservation, and link stability. The experimental result shows that C.R.C.P.R. improves network throughput and lifetime under controlled delay. 
Serhani et al. [21] designed an adaptive routing protocol (A.Q.) that relies on the reinforcement learning (R.L.) technique as a contribution to improving MANET-IoT data routing. The learning technique assesses the mobility of the nodes at a different time interval to identify the routing issues and adjust the routing metrics accordingly. Q-learning is augmented for adapting both dynamic and static network topologies. AQRL reduces transmission latency and improves delivery ratio consenting varying node mobility. In order to improve MANET performance optimization, intelligent water drops inspired routing protocol (I.W.D.R.P.) is designed by Sayad et al. [22].

\subsection{Adaptive multi-hop routing (A.M.R.)}

Multi-hop routing in MANET is dependent on the path nodes for successful packet delivery. Though there are many drawbacks in multi-hop routing and transmission, it is mandatory to evade the challenges in order to support real-time application scenarios. The capacity of buffer of a node is limited that reflects in the performance of MANET in terms of delay and packet delivery ratio. A.M.R. is designed to balance the end-to-end performance of MANET by adapting feasible neighbor selection solutions. Neighbor selection induces path selection that manages both limited buffer constraint and maximum throughput [23], [24].

\subsection{MANET model}

In this article, a random topology consisting of ' $n$ ' IoT nodes is modeled for estimation. The nodes pursue random movement patterns and communicate using defined bandwidth $\mathrm{B}$. The mobile IoT nodes possess a uniform communication range $\mathrm{c} \_\mathrm{r}$ and the nodes communicate in time slots $\left(\mathrm{s}_{\mathrm{t}}\right)$ allocated by the sender/receiver [25]-[27]. The rate of packet generation $\left(\emptyset_{\mathrm{g}}\right)$ is modeled as a Barnoulli representation for mapping packets in appropriate time slots. From the network representation, the objective of the routing is defined as in (1).

$$
\left.\begin{array}{c}
\sum d_{r}=\frac{B}{n \times s_{t}} \\
\text { such that } \\
\psi_{n}=\max d_{r} \forall \emptyset_{g} \in[0,1] \text { and } \\
\text { cost }=\min \left(\sum_{i=1}^{n} E\left(\emptyset_{g i}, i, t_{i}\right)\right)
\end{array}\right\}
$$

Here, $d_{r}, s_{t}, \psi_{n}$ represents data received, transmission sequence [28] and achievable $d r$ of $n$. The value of cost refers to the process required for offloading $\emptyset_{\mathrm{gi}}$ at time $t_{\mathrm{i}}$ to a neighbor node $i, i \in n$.

\section{METHOD}

MANET nodes possess a buffer with size $Z$. The occupancy rate $Z_{c}$ of the buffer is variable depending upon the $[\varnothing]_{\mathrm{g}}$ and $\mathrm{B}$ in the edge $\mathrm{E}$ between the nodes. An edge $\mathrm{E}$ is valid if dist (node 1, node 2$) \leq \mathrm{c} \_$. The probability of selecting a node for transmission $\mathrm{p}_{\mathrm{t}}$ relies $\llbracket \emptyset \rrbracket_{\mathrm{g}}$ and $\mathrm{Z}$ of a node. On the basis of the two factors, the $\llbracket \varnothing \rrbracket_{\mathrm{g}}$ is defined as:

$$
\emptyset_{g}=p_{t} \times \sum_{i=1}^{x} \frac{s_{t}-i}{t_{i}}
$$

Here, $x=Z$, if the received packets occupy the entire buffer. $\mathrm{o}<\mathrm{x}<\mathrm{Z}$, if the received packets is less than the buffer size.

In a congruent analysis, if $x>Z$, the buffer faces packet drop such neighbor loses its preference in neighbor selection. The design of the neighbor selection process is considered to adapt both $Z_{c}$ and $[\varnothing]_{g}$. Packet relaying follows a first-in-first-out queuing model where the pause time $\left(\mathrm{p}_{\mathrm{t}}\right)$ between two successive packets is maintained in a constant rate. This time varies with the packet drop $\left(\mathrm{p}_{\mathrm{d}}\right)$ and $\llbracket \emptyset \rrbracket_{\mathrm{g}}$ of the neighbor. High is the rate of $p_{d}$, less is the $\psi_{n}$ of a neighbor is computed using (3).

$$
\psi_{n}=\left\{\begin{array}{c}
\frac{1}{1-\phi_{g} / s_{t}} \times \frac{1}{\Delta e}, \text { if } Z_{c}=0 \\
\frac{1}{1-\frac{\phi_{g}}{s_{t}}} \times \frac{\alpha}{1-\frac{\phi_{g}}{\left(\emptyset_{g}+\emptyset_{d}\right)}} \times \frac{1}{\Delta e}, \text { if } 1 \leq Z_{c} \leq Z
\end{array}\right.
$$

Here, $\Delta \mathrm{e}$ represents the variation error of the packets in a buffer other than transmitted and dropped (i.e): 


$$
\left.\begin{array}{c}
\Delta e=\left(\emptyset_{g}+p_{d}-Z_{c}\right) \forall Z_{c} \neq 0 \text { and } \\
\propto=\frac{\emptyset_{g}\left[1-\frac{p_{d}}{\left(\emptyset_{g}+p_{d}\right)}\right]}{\frac{p_{d}}{\left(\varnothing_{g}+p_{d}\right)}\left[1-\frac{\emptyset_{g}}{\left(\emptyset_{g}+p_{d}\right.}\right]}
\end{array}\right\}
$$

From (3), it is seen that $\psi_{\mathrm{n}}$ holds two types of conditions for empty buffer and partially occupied buffer. In accordance with the objective in (1), the condition in (3) is analyzed. Increase in $\emptyset_{g}$ increases $\psi_{n}$, maximum $d_{r}$. In this case, $B$ is maximum utilized for $\forall \emptyset_{g}$. The analyses of the objective with the above conditions are described as follows.

Condition 1: if the buffer occupancy tends to zero (i.e.) $Z_{c}=0$.

Objective analysis: If the buffer occupancy of a node tends to zero, then $t_{p}$ between the packets is constant and also, $\emptyset_{g} \times t_{t}=\psi_{n} \forall t_{p} \leq t_{s}$. In a communication time slot $t_{s}$, if $\emptyset_{g} \times t_{t}=\psi_{n}$ is satisfied, then delivery delay $\left(t_{d}\right)$ is estimated as:

$$
t_{d}=t_{s}+t_{p}+\left(Z_{c}-s_{t}\right)\left(t_{t}-t_{r}\right)
$$

The factor $\left(Z_{c}-s_{t}\right) \times\left(t_{t}-t_{r}\right)$ refers to the packets generated in $t_{s}$ but required $t_{p}$ for transmitting the remaining $\left(Z_{c}-s_{t}\right)$ Packets. Here $t_{t}$ and $t_{r}$ are the transmits and receiving time of the packet. In this case, the packet is accepted by the node at a fixed time interval after dispatching a packet. Therefore,

$$
\sum d_{r}=\sum \psi_{n}+\frac{z_{c}}{n-2+Z_{c}}
$$

substitute for $\sum d_{r}$ from (1) and considering the path nodes (leaving out the source and destination),

$$
B=\frac{z_{c}}{z_{c}+k}, k=\text { a constant for }(n-2)
$$

From (7), the bandwidth of an Erelies on the capacity of the node buffer if $\psi_{n} s_{t}$ is a constant. If the order of packet arrival and dispatching in linear, the $s_{t}$ remains the same for all the $t_{i}$ is not required, and the cost $=0$. The transmission process if condition 1 is represented in Figure 1 .

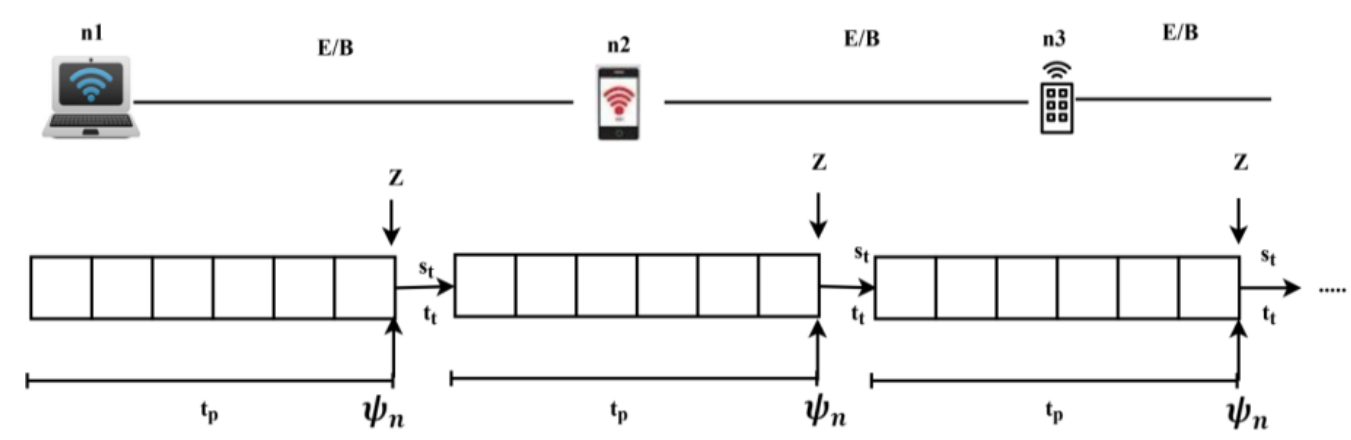

Figure 1. Transmission process

Condition 2: If the buffer occupancy of a node is greater than zero and less than the size of the buffer (i.e.) $1 \leq Z_{c} \leq Z$.

Objective analysis: This case occurs if $\emptyset_{g}$ of the nodes vary in an allocated $t_{s}$. Therefore $t_{p}$ of the packets in a buffer varies and hence $p_{d}$ is unavoidable. The objective in this condition is assessed under two primitives (i.e.) the rate of $t_{p}$ is high for the forwarder or the $t_{p}$ is high for the receiver. Both the constraints are analyzed for the path nodes leaning out the source and destination.

Constraints 1: $t_{p}$ for the sender is comparatively high than the receiver.

Analysis 1: If this constraint is achieved, the receiver experiences no $p_{d}$. Packet drop comes only if $\emptyset_{d}$ exceeds $Z_{c}$ of the receiver. Instead, the receiver experiences an underflow in the transmission interval difference time (i.e.) $\left(t_{p-l o w}-t_{p-\text { high }}\right)$. This time is augmented with delay factor and therefore, 


$$
t_{d}=t_{s}+\left(t_{p-l o w}-t_{p-h i g h}\right) \times s_{t}+\left(Z_{c}-s_{t}\right)\left(t_{t}-t_{r}\right)
$$

is the delay experienced. The factor $\left(t_{p-l o w}-t_{p-h i g h}\right)$ increases the number of communication time slot. If the consecutive time slot assignment demands allocation time, the enqueued packets are dropped. This means an offloading process is required in this condition. The throughput and cost in this case are:

$$
\left.\begin{array}{c}
\sum d_{r}=\sum \psi_{n} \times\left(\frac{p_{d}}{\emptyset_{g}}-\alpha\right)+\frac{z_{c} \times \Delta e}{(n-2)+z_{c}} \\
\text { and } \\
\cos t=\sum_{i=1}^{n} E\left[\left(\emptyset_{g}-\beta\right), i, t_{s+i}\right]
\end{array}\right\}
$$

where, $\beta=\left(t_{p-\text { low }}-t_{p-\text { high }}\right) \times s_{t}$

Constraint 2: $t_{p}$ for the sender is less compared to the receiving IoT node.

Analysis 2: The receiving IoT node dispatches its $Z$ packet at a prolonged time interval compared to the transmitting node. The rate of $\emptyset_{g}$ of the sender is high whereas $\psi_{n}$ of the receiver is less. Therefore, the $\sum d_{r}$ is obviously less. Offloading is in at most demand for balancing this constraint. The limited node capacity is utilized for effective transmission with the aid for offloading. In this constraint, throughput and cost is given by (10),

$$
\left.\begin{array}{c}
\sum d_{r}=\sum \psi_{n} \times\left(1-\sum\left(\frac{p_{d}}{\emptyset_{g}}\right)\right)+\frac{z_{c} \times\left(p_{d}\right)}{(n-2)+z_{c}} \\
\operatorname{cost}=\sum_{i=1}^{n} E\left[\left(\emptyset_{g}-\gamma\right), i, t_{p+i}\right]
\end{array}\right\}
$$

where, $\gamma=\frac{\psi_{n-r}}{\frac{\phi_{g-s} \times s_{t}}{d_{r}}}$, and $\psi_{n-r}$ is the achievable $d_{r}$ rate of the receiver. The delay observed here is:

$$
t_{d}=t_{s}+\left(t_{p-h i g h}-t_{p+i}\right) \times s_{t}+\left(Z_{c}-s_{t}\right)\left(t_{t}-t_{r}\right)
$$

The throughput observed in (6) is high compared to that in (9) and (10). Similarly, the delay in (5) is less compared to the delay in (8) and (11). Therefore offloading is augmented to the transmission/neighbor selection to achieve (1) in condition 2. The constraint 1 and 2 transmission process is illustrated in Figures 2(a) and 2(b) correspondingly.

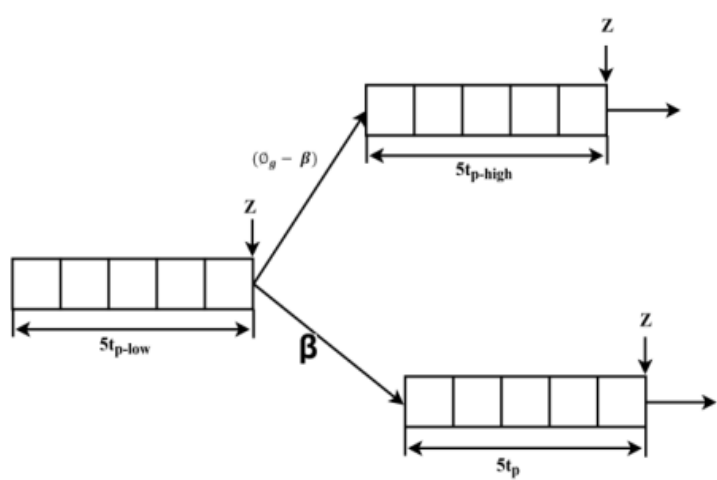

(a)

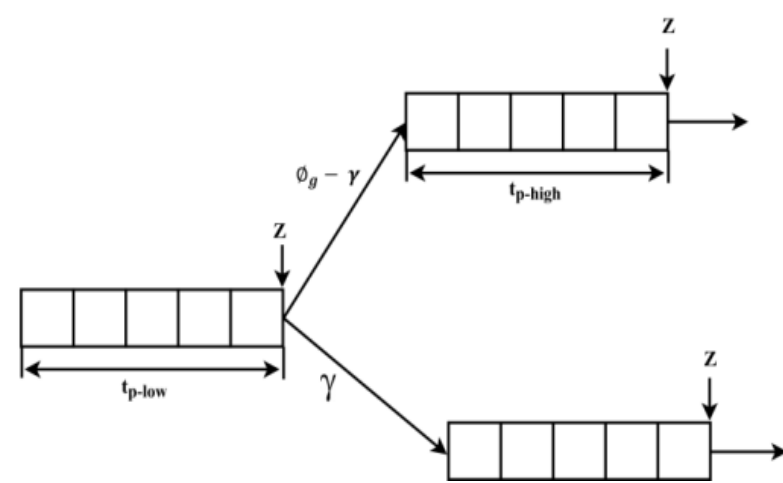

(b)

Figure 2. Transmission process; (a) constraint 1, (b) constraint 2

In the above summary, for offloading and non-offloading situations, the conditions and performance analysis are classified. A.M.R. selects capacity built nodes for handling packets along the transmission, as a multi-hop routing. In terms of the increasing network density and traffic load, this helps to balance latency and network throughput. A.M.R. specifically selects neighbours with secure offloading features capable of dispatching traffic loads. The following section explains the process of routing, collection of neighbours and offloading. 


\subsection{Routing and neighbor selection}

A conventional multi-hop routing protocol operated from the network layer of the IoT node. Routing and transmission are segregated between ad-hoc and IoT layers of the network. Figure 3 portrays a schematic representation of MANET-IoT network.

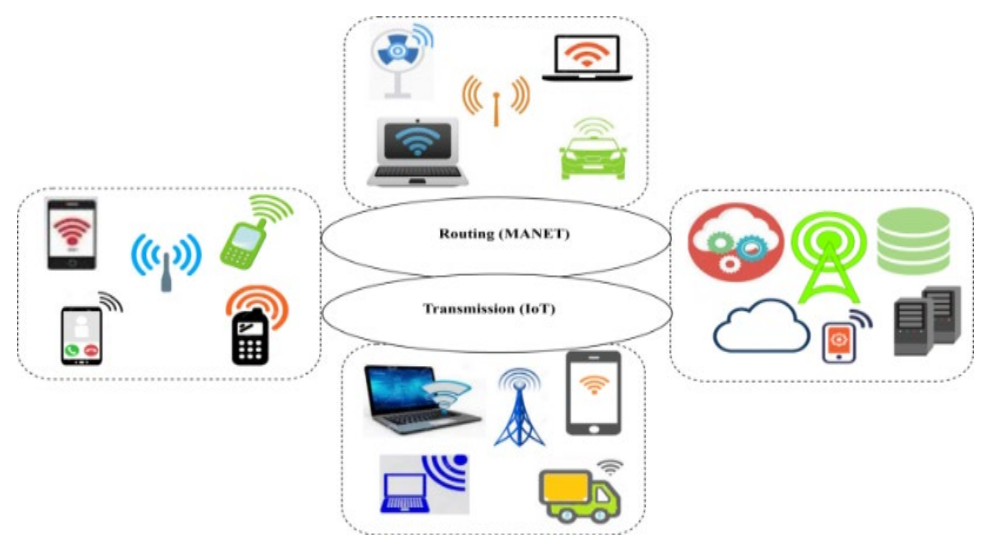

Figure 3. MANET-IoT representation

It is the task of MANET routing protocols to discover routes between the sender and receiver. Using the concept of IoT communication, packet data sharing, and offloading are facilitated. To achieve better network performance, both primitives are harmonized to achieve un-biasing node capacity and delay. The sender chooses the shortest distance node for packet exchange, as per the traditional routing method. By changing the transmission rate based on node power, the preferred route is effectively maintained for a prolonged period. This helps manage variable data traffic packets and modifications to limited routes. The sender generates varying $\emptyset_{g}$ depending on the application, it is supporting. In a normalized routing, the fault routes are replaced by the next available nodes estimated using a distance metric. This causes additional control messages, increasing neighbor selection, and communication costs.

The offloading process that is naive propriety of IoT is harmonized for improving the packet handling capacity of the route nodes. This prevents frequent path switch-over and neighbor selection and retains path stability. The previously discussed conditions and constraints are considered for offloading data packets. The rate of offloading varies in constraint 1 and 2 such that $\beta$ and $\gamma$ are the packets to be offloading respectively. The cost as described in (1) is expected to be less to perform offloading. The cost is defined using (12).

$$
\operatorname{cost}=w_{1} \times t_{d}+w_{2}+s_{t}
$$

Where $w_{1}$ and $w_{2}$ are the adjustable weights for delay and transmission sequence. The factor $t_{d}$ is elaborated on the basis of $\psi_{n}$ of an IoT node. An IoT scenario exhibits both device-to-device and device-toinfrastructure packet exchange. The $t_{d}$ relies on the packet's acceptance and $Z_{c}$ of the receiving node/infrastructure. Therefore, the offloading cost $\left(\operatorname{cost}_{\text {off }}\right)$ is estimated as:

$$
\operatorname{cost}_{o f f}=\sum_{i=1}^{Z} \frac{\operatorname{cost}_{i}}{Z_{c i}+t_{t i}}
$$

From (12), if the cost is less, then $\operatorname{cost}_{\text {off }}$ is less. Similarly, the offloading cost of an infrastructure is given with the consideration of $B$ using (14).

$$
\cos _{o f f}=\sum_{i=1}^{Z} \frac{\operatorname{cost}_{i}}{Z_{c i}+t_{t i}}+\frac{\sum_{i=1} \phi_{g i} \times \frac{1}{t_{t i}}}{B}
$$

Here, $B$ is fixed for all the $E$ in the network. Now, the offloading is performed for the constraints discussed under condition 2 . The case discussed in condition 1 is ideal and it does not require any offloading Route swapping/new path discovery is required if the communicating IoT is unavailable. The constraints 1 and 2 under condition 2 require offloading at specified time intervals. This process is discussed below. 


\subsection{Offloading process for constraint 1}

For the condition discussed in constraint 1 , the time delay required is estimated using (8). In this delay, the offloading is performed for $\beta$ time (i.e) $\left(t_{p-l o w}-t_{p-h i g h}\right) \times s_{t}$. The offloading process is initiated by establishing $E$ concurrently with an IoT node that is present in the $c_{r}$ of the sensor. The neighbor handles packets for a time period of $\left(t_{p-\text { low }}-t_{p-h i g h}\right) \times s_{t}+\left(Z_{c}-s_{t}\right) \times\left(t_{t}-t_{r}\right)$. Here, as two nodes are concurrently selected, the time delay is given by;

$$
t_{d}=t_{s}+\left(t_{p-l o w}-t_{p-h i g h}\right) \times s_{t}+\sum_{i=s_{t}}^{Z_{c}}\left(t_{t}-t_{r}\right)
$$

The first quadrant in (15) is valid until $t_{s}$ where in $\sum_{i=s_{t}}^{Z_{c}}\left(t_{t}-t_{r}\right)$ is zero. Therefore, selecting a new neighbor for concurrent offloading generates a delay of $t_{d}(5)-\sum_{i=s_{t}}^{Z_{c}}\left(t_{t}-t_{r}\right)$ for handling $\left(Z_{c}-\right.$ $s_{t}$ ) packets. The cost for offloading is then derived as:

$$
\operatorname{cost}_{\text {off }}=\left\{\begin{array}{c}
\frac{w_{1} \times\left(t_{t}-t_{r}\right) \times\left(Z_{c}-s_{t}\right)}{\left(Z_{c}-s_{t}\right)+\left(t_{s}-\frac{\beta}{s_{t}}\right)} \text {, if the neighbor is an IoT node } \\
\frac{w_{1} \times\left(t_{t}-t_{r}\right) \times\left(Z_{c}-s_{t}\right)}{\left(Z_{c}-s_{t}\right)+\left(t_{s}-\frac{\beta}{s_{t}}\right)}+\frac{\sum_{i=s_{t}}^{Z_{c}} \emptyset_{g i} \times \frac{1}{t_{t i}}}{B}, \text { if the neighbor is an Infrastucture }
\end{array}\right.
$$

The offloading cost presented in (13) and (14) are estimated for the entire size of the buffer. This case avoided by classifying offloading and non-offloading packets based on $\emptyset_{g}$ and $\psi_{n}$. T or the buffered packets $\mathrm{Z}$, offloading is performed for $\beta$ as illustrated in Figure 2(a).

\subsection{Offloading process for constraint 2}

This condition is different from the previous case as the time required for an active concurrent neighbor is high. The offloading time required is $\left(t_{p-h i g h}+t_{p+i}\right) \times s_{t} \forall \gamma$. This time is alternatively set between the sender and receiver. Let the $t_{s}$ be split into different $t_{t}$ an instance such that $\Delta \cdot t_{p-s}=t_{p-r}$ is the actual pause time, $t_{p-s}$ and $t_{p-r}$ are the pause time of the sender and receiver and $\Delta$ is the frequency of the alternate offloading phase. The $t_{s}$ is now spilt into $\Delta . t_{t}$ time instances such that offloading occurs in $\left\{\frac{t_{s}}{\Delta t_{t}}, \frac{t_{s}}{2 \Delta t_{t}}, \ldots \frac{t_{s}}{z \Delta t_{t}}\right\}$. The times delay hence here is modeled as

$$
t_{d}=t_{s}+\sum_{j=1}^{Z}\left(Z_{c}-s_{t}\right) \times\left(t_{t}-t_{r}\right)+\left(\Delta_{j}-\Delta_{j-1}\right)
$$

Unlike the previous case, in (17), $t_{s}$ is estimated for 1 to $Z$ and $\sum_{j=1}^{Z}\left(Z_{c}-s_{t}\right) \times\left(t_{t}-t_{r}\right)+\left(\Delta_{j}-\Delta_{j-1}\right)$ is estimated for $\frac{t_{s}}{\Delta}$ Occurrences. The offloading cost is then reduced to

$$
\operatorname{cost}_{o f f}=\left\{\begin{array}{c}
\frac{w_{1} \times\left(t_{t}-t_{r}\right) \times \frac{t_{s}}{\Delta}}{Z+\left(t_{s}-\gamma \times s_{t}\right)}, \text { if the neighbor is an IoT node } \\
\frac{w_{1} \times\left(t_{t}-t_{r}\right) \times \frac{t_{s}}{\Delta}}{Z+\left(t_{s}-\gamma \times s_{t}\right)},+\frac{\sum_{j=1}^{Z} \emptyset_{g j} \times \frac{1}{\left(\Delta_{j}-\Delta_{j i}\right)}+\left(t_{t j}-t_{r j}\right)}{B}, \text { if the neighbor is an infrastucture }
\end{array}\right.
$$

A.M.R. sustains routing and transmission by classifying the capacity of the neighbors as per conditions 1 and 2. The remitting constraints under condition 2 are experienced by the route node under varying transmission load. Therefore, the estimation of delay and cost, as represented in the offloading process, is estimated to choose neighbors satisfying the offloading process as estimated above. The process is unanimous for all the path nodes that constitute the multi-hop route to the destination. More specifically, A.M.R. is adaptive in accepting path nodes as estimated by their offloading capacity for handling varying traffic load. The nodes opted for packet data handling, and transmission is seamlessly evaluated for the buffer constraints and delay model, so optimized aid performance irrespective of the network density and traffic load.

\section{PERFORMANCE EVALUATION}

AMR functions are implemented in a MANET scenario generated using network simulator-3. The network is placed with 150 MANET and IoT nodes that perform both device-to-device and device-toinfrastructure communications. Table 1 presents a detailed description of the simulation parameters and 
configuration. Using a comparative analysis for the metrics performance, packet delivery ratio, delay, offloading latency, packet acceptance, packet drop, and overhead, A.M.R. reliability is verified. Existing A.O.D.V.C.S. [29], SynAnt [19], and AQ-RL [21] are considered for the comparative analysis along with the proposed A.M.R.

Table 1. Simulation parameters and configuration

\begin{tabular}{cc}
\hline Simulation Parameter & Configuration \\
\hline Number of Nodes & 150 \\
Number of Infrastructure Units & 4 \\
MANET Topology Dimensions & $750 \mathrm{~m} \times 500 \mathrm{~m}$ \\
Packet Size & 1024 bytes \\
Traffic Class & Constant Bit Rate \\
B & $10 \mathrm{Mbps}$ \\
Buffer Capacity & 50 data packets \\
Maximum Transmission Flows & 10 \\
$t_{p}$ & $0-600 \mathrm{~ms}$ \\
\hline
\end{tabular}

\subsection{Throughput analysis}

A comparative study of network throughput concerning the traffic load is presented in Figure 4. In A.M.R., the reliable neighbors for packet handling are selected by pre-estimating the offloading constraints. The rate of $\psi_{n}$ in an ideal transmission, the condition is deliberated by evaluating $\emptyset_{g} \times t_{t}$ withint $s_{s}$. Similarly, in the exceeding constraint 1 and 2 (if experienced by a node), $\sum d_{r}$ is determined by the normalized path node and offloading node. Hence, the rate of $\psi_{n}$ is retained by the $\emptyset_{g}$ of two or more neighbors achieving $\max d_{r}$. In constraint $2, \emptyset_{g}$ is achieved by selecting a neighbor with a maximum $\psi_{n-r}$. The received packets observed in $t_{s}\left(t_{t}-t_{r}\right)$ and $t_{p+i}$ intervals are successful with fewer drops due to route and offloading nodes in the transmission path. In both the routes, the achieved throughput is high.

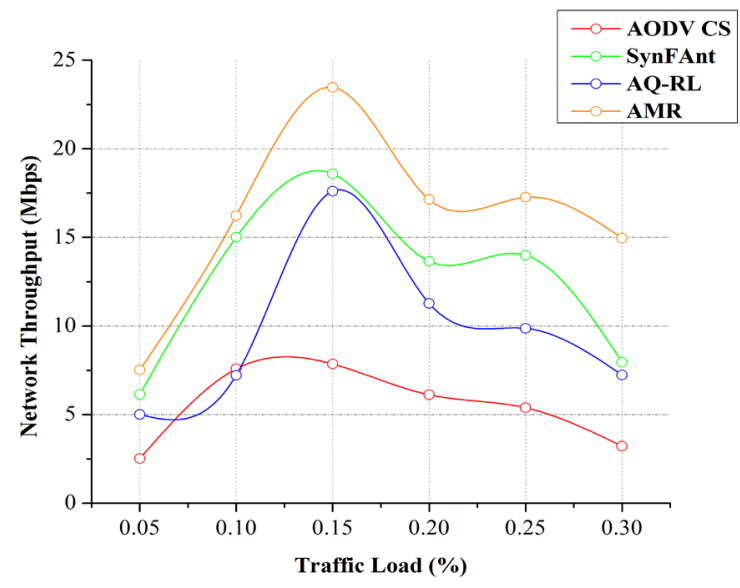

Figure 4. Throughput comparisons

\subsection{Delivery ratio analysis}

Figure 5, presents the comparative analysis of packet delivery ratio between the existing and proposed methods. The rate of packet transmission in A.M.R. is verified using $\psi_{n}$ for all $\left(1-\frac{p_{d}}{\emptyset_{g}}\right)$ packets from the intermediates. The time intervals $t_{s}$ and $t_{p+i}$ maximizes $\left(1-\frac{p_{d}}{\emptyset_{g}}\right)$ and $\left(Z_{c}-s_{t}\right) \times\left(t_{t}-t_{r}\right)$ packets at the destination. Therefore the ratio between $\left(1-\frac{p_{d}}{\emptyset_{g}}\right)+\left(Z_{c}-s_{t}\right) \times\left(t_{t}-t_{r}\right)$ and $\varphi_{n}$ of the source is high in all transmission intervals, retaining the packet delivery ratio of the network.

\subsection{Delay analysis}

An increase in node density requires an additional routing procedure, and hence the delay increases. In A.M.R., delay due to neighbor discovery and packet re-transmission is confined at the initial state. The 
nodes facing trivial transmission constraints are identified [30], [31] and are provided with neighbors to avoid unnecessary packet overflow and congestion [32]. The delay estimated due to varying traffic flow using (5), (8), and (11) is controlled by selecting cost-efficient neighbors. Therefore, the $t_{d} /$ nodes is the average reduced delay as estimated using (15) and (17). The delay estimated in (5) is an ideal case and cannot be reduced. More specifically, offloading controls $\left(t_{p+i}+t_{p-h i g h}\right)$ that reduces delay in the proposed A.M.R. shown in Figure 6.

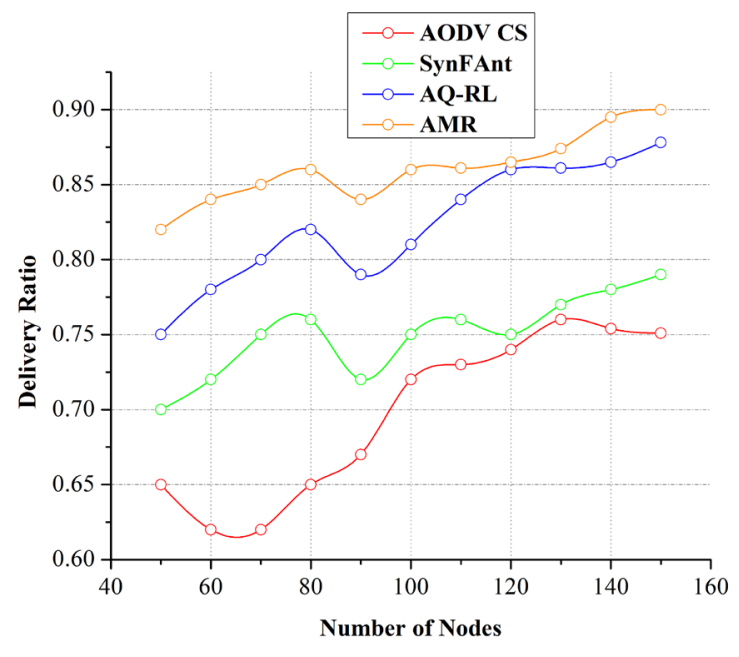

Figure 5. Delivery ratio comparisons

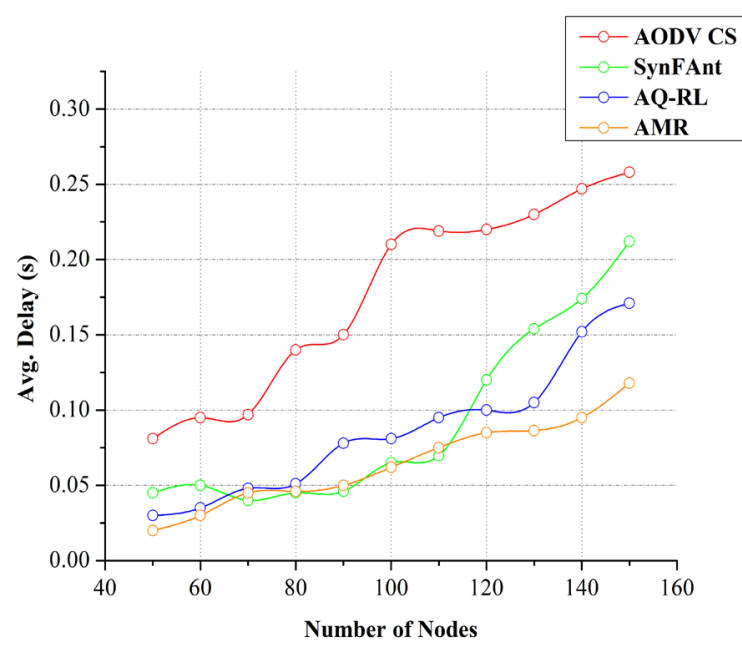

Figure 6. Delay comparisons

\subsection{Offloading latency analysis}

Offloading latency experienced in the proposed A.M.R. is compared with the existing methods in Figure 7. With respect to the varying pause time, the nodes in the network are categorized as having the

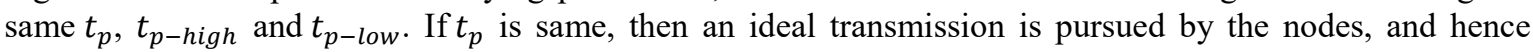
offloading is not necessary. In a scenario if $t_{p-h i g h}$ or $t_{p-l o w}$ is experienced, the offloading time required is $\left(t_{p+i}-t_{s}\right)$ (for constraint 1$)$ and $\left(\Delta_{j}-\Delta_{j-1}\right)$ (for constraint 2), respectively. If the offloading process extends to the second $t_{s}$, then $\left(\Delta_{j}-\Delta_{j-1}\right)$ and $\left(t_{p+i}-t_{s}\right)$ is estimated within $2 t_{s}$ else it is computed within the first $t_{s}$. In both cases, the process of offloading and transmission is concurrently performed. Therefore, the time for offloading is consistently less compared to packet re-transmission time.

\subsection{Packet acceptance analysis}

The rate of packet acceptance with respect to the number of flows is portrayed in Figure 8 . The varying traffic flow increases the number of packets handled by the path nodes. This increases the rate of congestion and buffer overflow. The process of offloading is instigated at regular overflow intervals such as $t_{p+i}$ and $t_{p-h i g h}+t_{p+i}$ to improve the rate of packet queuing and dispatching. The remaining $\left(Z_{c}-\right.$ $s_{t}$ ) packets are offloaded of the transmission is not in an ideal state. This helps to prevent buffer level overloading and retains the acceptance rate of the packets at $\left\{\frac{t_{s}}{\Delta t_{t}}, \frac{t_{s}}{2 \Delta t_{t}}, \ldots \frac{t_{s}}{Z \Delta t_{t}}\right\}$ intervals. As the $\mathrm{Z}$ exceeding packets are estimated based on the bandwidth usage and generation rate of the nodes, the neighbor with appropriate $Z_{c}$ capacity is selected for handling $\left(Z_{c}-s_{t}\right)$ packets. This helps A.M.R. to retain a high packet acceptance rate.

\subsection{Packet drop analysis}

The number of packet drop accounted for here is with reference to the overflow packets exceeding $\mathrm{Z}$ of a node. If the offloading process is sequential, packet drop is prevented. Instead, in the constraint 1 and two, there are two instances for packet drops i.e. $t_{p+i}$ and $\left(t_{s}+\Delta_{j}\right)$. In these cases, if the offloading time is high, then the fore-mentioned interval experiences packet drop. The offloading time of A.M.R. is confined to $\left(\Delta_{j}-\Delta_{j-1}\right)$ and $\left(t_{p+i}-t_{s}\right)$, respectively, and therefore, the packet drop experienced in this new interval is less compared to the interval estimated before. The observed packet drop in every time slot is depicted in Figure 9. 


\subsection{Overhead analysis}

Figure 10 presents the comparative analysis of overhead between the existing and proposed methods. Additional route discovery and control message exchange due to frequent path switch-over are less in A.M.R. The packet overflow is reduced by balancing node capacity and delay exploiting the offloading feature of IoT in MANET nodes. The buffer optimization and packet handling at every hop are equally prioritized for improving the transmission rate, and hence the network outcome. Cost based offloading assists in choosing a reliable neighbor active for a prolonged time. Therefore, the need for an alternate neighbor or route within a specific $t_{s}$ is less, requiring less number of control messages. This retains data packet flow over the control messages reducing overhead. In Table 2, the values of the comparative analysis are presented. Based on the discussions, the proposed A.M.R. method attains better throughput (14.16 Mbps), delay (0.118), packet drop (126), and overhead (36 packets) when compared to existing methods such as AODC CS, SynFAnt, AQ-RL.

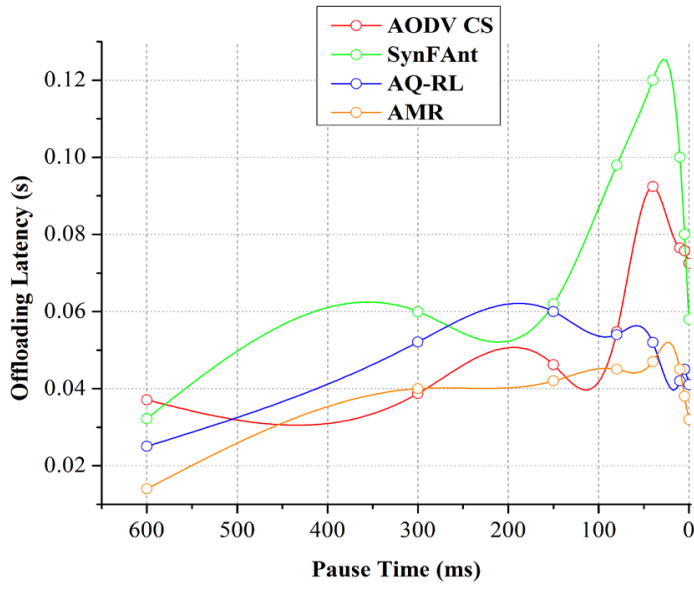

Figure 7. Offloading latency comparisons

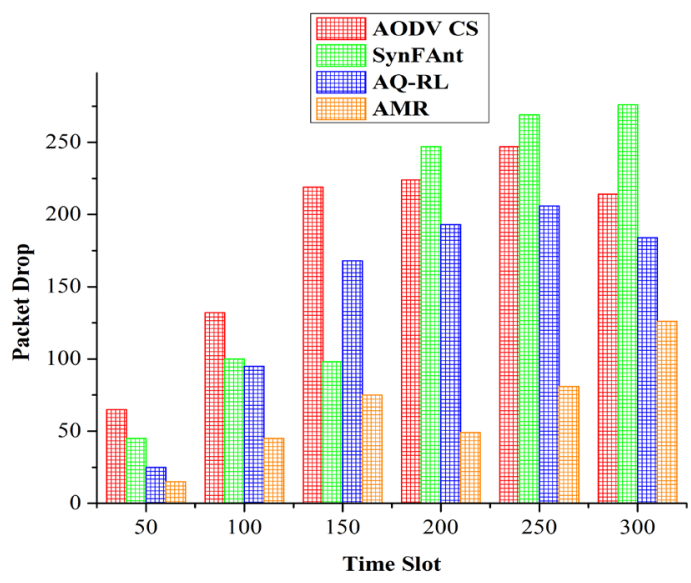

Figure 9. Packet drop comparisons

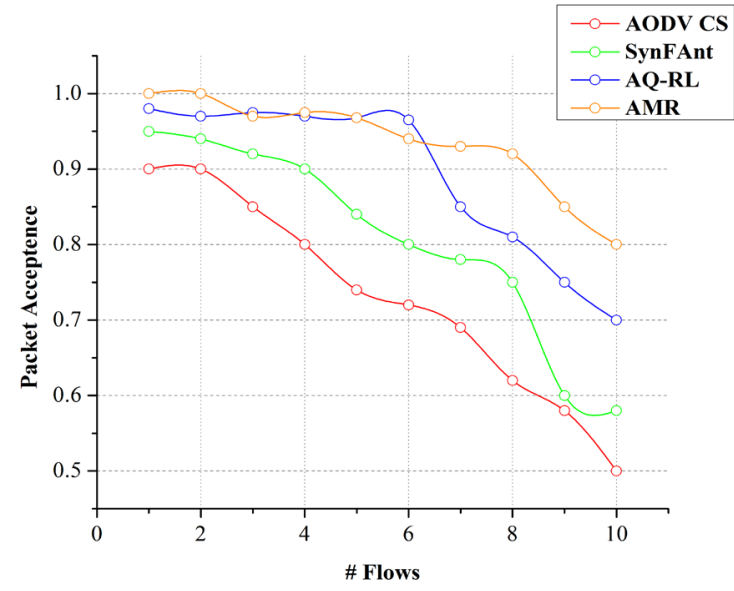

Figure 8. Packet acceptance comparisons

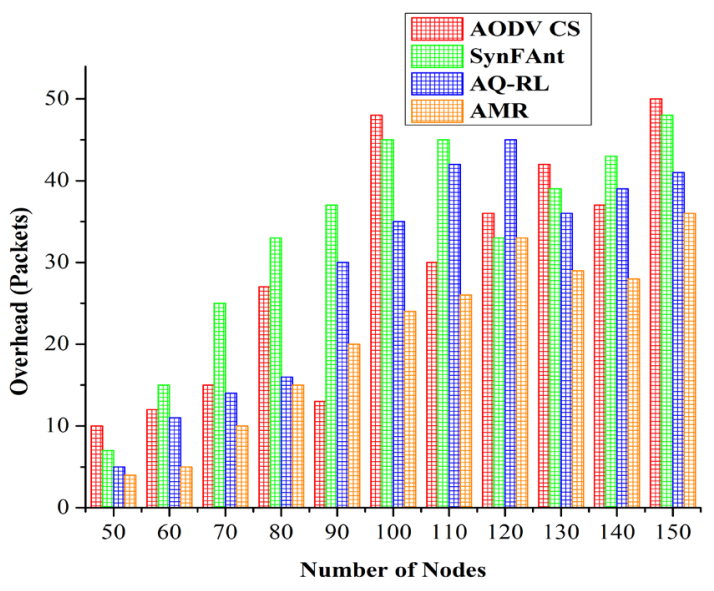

Figure 10. Overhead comparisons

Table 2. Comparative analysis values

\begin{tabular}{ccccc}
\hline Metric & AODV CS & SynFAnt & AQ-RL & AMR \\
\hline Throughput (Mbps) & 3.21 & 7.95 & 7.23 & 14.96 \\
Delivery Ratio & 0.751 & 0.79 & 0.878 & 0.9 \\
Avg. Delay (s) & 0.258 & 0.212 & 0.171 & 0.118 \\
Offloading Latency (s) & 0.0371 & 0.0322 & 0.025 & 0.014 \\
Packet Acceptance & 0.5 & 0.58 & 0.7 & 0.8 \\
Packet Drop & 214 & 276 & 184 & 126 \\
Overhead (Packets) & 50 & 48 & 41 & 36 \\
\hline
\end{tabular}




\section{CONCLUSION}

This manuscript discusses adaptive multi-hop routing designed for MANET-IoT networks. A.M.R. balances the trade-off between node capacity and delays to levitate network throughput. The initial routing constraints connected to packet handling and transmission are mitigated by identifying optimal neighbors that support offloading. Transmission constraints are mitigated by selecting reliable neighbors estimated using an offloading cost to achieve better network throughput under controlled delay. The proposed A.M.R. performs unanimous offloading for all the nodes in each hop to reduce overhead and packet drop with an improved delivery ratio.

\section{REFERENCES}

[1] Asha and G. Mahadevan, "An adaptive cross-layer architecture to optimize qos provisioning in manet," Indonesian Journal of Electrical Engineering and Computer Science, vol. 6, no. 1, pp. 16-25, Apr. 2017, doi: 10.11591/ijeecs.v6.i1.pp16-25.

[2] M. L. Mutar, B. M. Aboobaider, and A. S. Hameed, "Review paper in vehicle routing problem and future research trend," Int. J. Appl. Eng. Res., vol. 12, no. 22, pp. 12279-12283, 2017.

[3] F. Forero R., N. M. Peña T., and N. L.S. da Fonseca, "Geometric aspects of probabilistic broadcasting in ad hoc networks," Ad Hoc Networks, vol. 87, pp. 146-156, 2019, doi: 10.1016/j.adhoc.2018.11.014.

[4] H. Bin Liaqat, A. Ali, J. Qadir, A. K. Bashir, M. Bilal, and F. Majeed, "Socially-aware congestion control in ad-hoc networks: Current status and the way forward," Futur. Gener. Comput. Syst., vol. 97, pp. 634-660, 2019, doi: 10.1016/j.future.2019.02.017.

[5] A. A. J. Mohammed, M. A. Burhanuddin, and H. Basiron, "Key Enablers of IoT Strategies in the Context of Smart City Innovation,” Jour Adv Res. Dyn. Control Syst., vol. 10, no. 04, pp. 582-589, 2018.

[6] J. Favela, M. Rodriguez, M. Alba, and A. L. Morán, "Supporting Opportunistic Interactions with People, Resources and Agents in Ubiquitous Enviroments," in Mobile Ad-hoc Collaboration Workshop at CHI 2002, 2002.

[7] P. M. Shakeel, S. Baskar, and S. Selvakumar, "Retrieving Multiple Patient Information by Using the Virtual MIMO and Path Beacon in Wireless Body Area Network," Wirel. Pers. Commun., vol. 108, pp. 2359-2370, 2019, doi: 10.1016/j.adhoc.2018.11.014.

[8] S. K. S. L. Preeth, R. Dhanalakshmi, R. Kumar, and P. M. Shakeel, "An adaptive fuzzy rule based energy efficient clustering and immune-inspired routing protocol for WSN-assisted IoT system," J. Ambient Intell. Humaniz. Comput., 2018, doi: 10.1007/s12652-018-1154-z.

[9] Prasad A. Y. and R. Balakrishna, "Implementation of optimal solution for network lifetime and energy consumption metrics using improved energy efficient LEACH protocol in MANET," TELKOMNIKA (Telecommunication, Computing, Electronics and Control), vol. 17, no. 4, pp. 1758-1766, Aug. 2019, doi: 10.12928/TELKOMNIKA.v17i4.12004.

[10] M. H. Hassan et al., "A general framework of genetic multi-agent routing protocol for improving the performance of manet environment," IAES Int. J. Artif. Intell., vol. 9, no. 2, pp. 310-316, Jun. 2020, doi: 10.11591/ijai.v9.i2.pp310-316.

[11] I. A. Alameri, "MANETS and Internet of Things: The Development of a Data Routing Algorithm," Eng. Technol. Appl. Sci. Res., vol. 8, no. 1, pp. 2604-2608, Feb. 2018.

[12] M. S. Talib, A. Hassan, Z. A. Abas, M. F. Ali, M. N. Al-Mhiqani, and A. A. Mohammed, "Clustering in VANETs perspective: Concepts, topology and applications," Int. J. Adv. Sci. Technol., vol. 28, no. 8, pp. 471-484, 2019.

[13] Y. H. Chen, C. C. Hu, E. H. K. Wu, S. M. Chuang, and G. H. Chen, "A Delay-Sensitive Multicast Protocol for Network Capacity Enhancement in Multirate MANETs," IEEE Syst. J., vol. 12, no. 1, pp. 926-937, Mar. 2018, doi: 10.1109/JSYST.2017.2677952.

[14] J. Li, B. N. Silva, M. Diyan, Z. Cao, and K. Han, "A clustering based routing algorithm in IoT aware Wireless Mesh Networks,” Sustain. Cities Soc., vol. 40, pp. 657-666, 2018, doi: 10.1016/j.scs.2018.02.017.

[15] D. Kang, H. S. Kim, C. Joo, and S. Bahk, "ORGMA: Reliable opportunistic routing with gradient forwarding for MANETs,” Comput. Networks, vol. 131, pp. 52-64, 2018, doi: 10.1016/j.comnet.2017.12.001

[16] M. S. Talib, A. Hassan, Z. Abas, A. A. H. Hassan, M. Ali, and Z. J. Al-Araji, "Clustering based affinity propagation in VANETs: Taxonomy and opportunity of research," Int. J. Recent Technol. Eng., vol. 7, no. 6, pp. 672-679, 2019.

[17] M. Zhang, M. Yang, Q. Wu, R. Zheng, and J. Zhu, "Smart perception and autonomic optimization: A novel bioinspired hybrid routing protocol for MANETs," Futur. Gener. Comput. Syst., vol. 81, pp. 505-513, 2018, doi: 10.1016/j.future.2017.07.030.

[18] I. Kacem, B. Sait, S. Mekhilef, and N. Sabeur, "A New Routing Approach for Mobile Ad Hoc Systems Based on Fuzzy Petri Nets and Ant System," IEEE Access, vol. 6, pp. 65705-65720, 2018, doi: 10.1109/ACCESS.2018.2878145.

[19] J. Bai, Y. Sun, C. Phillips, and Y. Cao, "Toward Constructive Relay-Based Cooperative Routing in MANETs," IEEE Syst. J., vol. 12, no. 2, pp. 1743-1754, 2018, doi: 10.1109/JSYST.2017.2721543.

[20] A. A. M. Al-Najjar and H. S. Chasib, "Design and implementation weights equation for optimization DSR protocol in MANETs environment," Int. J. Adv. Sci. Technol., vol. 28, no. 8, pp. 457-470, 2019.

[21] A. Serhani, N. Naja, and A. Jamali, "AQ-Routing: mobility-, stability-aware adaptive routing protocol for data routing in MANET-IoT systems," Cluster Comput., vol. 23, pp. 13-27, 2020, doi: 10.1007/s10586-019-02937-x. 
[22] L. Sayad, L. Bouallouche-Medjkoune, and D. Aissani, "IWDRP: An Intelligent Water Drops Inspired Routing Protocol for Mobile Ad Hoc Networks," Wirel. Pers. Commun., vol. 94, no. 4, pp. 2561-2581, 2017, doi: 10.1007/s11277-016-3692-z.

[23] I. Takeuchi and M. Sugiyama, "Target neighbor consistent feature weighting for nearest neighbor classification," in Advances in Neural Information Processing Systems 24: 25th Annual Conference on Neural Information Processing Systems 2011 (NIPS 2011), 2011, pp. 1-17.

[24] P. M. Shafi, S. Selvakumar, and P. M. Shakeel, "An efficient optimal fuzzy C means (OFCM) algorithm with particle swarm optimization (PSO) to analyze and predict crime data," J. Adv. Res. Dyn. Control Syst., vol. 6, pp. 699-707, 2018.

[25] P. Bellavista, G. Cardone, A. Corradi, and L. Foschini, "Convergence of MANET and WSN in IoT urban scenarios," IEEE Sens. J., vol. 13, no. 10, pp. 3558-3567, 2013, doi: 10.1109/JSEN.2013.2272099.

[26] N. binti Harum, M. F. Ali, N. A. Zakaria, and S. Anawar, "Smart surveillance system using background subtraction technique in IoT application," Int. J. Adv. Comput. Sci. Appl., vol. 9, no. 12, pp. 122-128, 2018.

[27] M. F. Ali, N. Harum, N. A. Abu, M. N. Al-Mhiqani, M. S. Talib, and A. A. Mohammed, "Protecting IoT based transmitted data security using tokenized multiple layered encryption techniques," Int. J. Adv. Sci. Technol., vol. 28, no. 8, pp 485-505, 2019.

[28] K. L. M. Ang and J. K. P. Seng, "Application Specific Internet of Things (ASIoTs): Taxonomy, Applications, Use Case and Future Directions," IEEE Access, vol. 7, pp. 56577-56590, 2019, doi: 10.1109/ACCESS.2019.2907793.

[29] A. Kout, S. Labed, S. Chikhi, and E. B. Bourennane, "AODVCS, a new bio-inspired routing protocol based on cuckoo search algorithm for mobile ad hoc networks," Wirel. Networks, vol. 24, pp. 2509-2519, 2018, doi: 10.1007/s11276-017-1485-2.

[30] N. Javaid, U. Shakeel, A. Ahmad, N. Alrajeh, Z. A. Khan, and N. Guizani, "DRADS: depth and reliability aware delay sensitive cooperative routing for underwater wireless sensor networks," Wirel. Networks, vol. 25, no. 11, pp. 777-789, 2019, doi: 10.1007/s11276-017-1591-1.

[31] S. Redhu, M. Anupam, and R. M. Hegde, "Optimal Relay Node Selection for Robust Data Forwarding over TimeVarying IoT Networks," IEEE Trans. Veh. Technol., vol. 68, no. 9, pp. 9178-9190, 2019, doi: 10.1109/TVT.2019.2929856.

[32] A. A. M. Al-Najjar, "Optimizing MANETs Network Lifetime Using a Proactive Clustering Algorithm," Turkish J. Comput. Math. Educ., vol. 12, no. 8, pp. 143-155, 2021, doi: 10.17762/turcomat.v12i8.2758. 